\title{
Increase of the Overturning Immunity of an Electric Tricycle
}

\author{
Alfred Pavlik, Jan Dizo, Pavol Kurcik, Miroslav Blatnicky, Peter Strazovec \\ Faculty of Mechanical Engineering, University of Žilina. Univerzitná 8215/1, 01026 Žilina. Slovak Republic. E-mail: \\ alfred.pavlik@fstroj.uniza.sk, jan.dizo@fstroj.uniza.sk,_ pavol.kurcik@fstroj.uniza.sk, miroslav.blat-
} nicky@fstroj.uniza.sk, peter.strazovec.@fstroj.uniza.sk

The main topic of this paper is the presentation of the keynote of implementation of the newly designed steering mechanism for a tricycle with the electric powertrain with two wheels on the rear axle. On the present, threewheeled road vehicles with two wheels on the rear axle are wholly dependent on the standard steering mechanism, which main task is the rotation of a front wheel about the steering axis in order to ensure the change of direction in curves. These vehicles are characterised by the relatively poor overturning immunity in a curve. Therefore, in our workplace the new steering mechanism was developed, which is intended to be mounted just in such threewheeled vehicles construction. The main principle feature of our innovative steering mechanism consists in the additional lateral movement of the front wheel of a vehicle, when it is driving in a curve. Thus, a tricycle using this newly designed steering system can run in curves at greater speed and driving is safer. In this paper, there are presented the working principle of the newly designed steering system as well as dynamic analyses of a threewheeled vehicle when it is running in curves.

Keywords: Three-wheeled vehicle, Dynamic simulation, Steering mechanism, Overturning immunity

\section{Introduction}

Constantly increased numbers of vehicles in larger or also smaller cities as well as the trend of prices of standard hydrocarbon fuels force car companies to orient they development on more and more fuel efficient and ecological vehicles. Despite of same exceptions, standard four-wheeled vehicles dominate on the automotive market. These vehicles are most often designed four or five passengers with additional luggage space. But, such a vehicles design limits significant improvements of the fuel efficiency.

On the other hand, we can see in urban operation small vehicles, such as bicycles, mopeds and motorcycles, which are more ecological from the consumed energy point of view. However, these two-wheeled vehicles are primary designed for one single passenger and only for delimited small amount of luggage. Hereby, they are not able to protect passengers from bad weather conditions on desired form.

Therefore, designers and engineers of transport means make the great effort to find effective way, how to increase consumption of standard hydrocarbon fuels of road vehicles $[1,2]$, but also of rail vehicles and others. There are many research focused on fuels saving, such as using alternative fuels, combination of several sources of energy know as hybrid powertrains $[6,30]$. Some of these approaches are more effective and but some of them still meet associated problems, such as negative effects from durability point of view, operating behaviours etc. Application of an electric powertrain for smaller urban vehicles seems to be very effective way for reducing consumption of hydrocarbon fuels and also air pollution in cities [5, 25].

Based on analysed facts mentioned above, our development and research team have decided to develop and design a smaller urban three-wheeled vehicle, which will joint (mortis) two main key factors, namely ecological electric powertrain and a new innovative steering mechanism improving overturning immunity.

\section{Technical solution of the newly designed stee- ring mechanism}

If we consider three-wheeled vehicles, we can divide into two categories: with two wheels on the front axle and with two wheels on the rear axle. Both have their advantages and also disadvantages.

In our project of the design of a smaller urban threewheeled vehicle with electric powertrain we have decided to become attached to the conception of a three-wheeled vehicle with two wheels on a rear axle.

The essential disadvantage of such a wheels layout is relatively poor overturning immunity when it is run in curves. It becomes evident mainly in curves with small radius at higher speeds. Therefore in our workplace there have arisen the fundamental task, how to eliminate this real negative feature of a standard steering mechanism. The effort has eventuated to the completely new technical solution of a steering mechanism of a three-wheeled vehicle [5].

Hence the main task of this paper is the presentation of principle features of our technical solution and also the comparison of driving properties of a three-wheeled vehicle with two wheels on a rear axle with a standard steering mechanism and with the new designed steering mechanism. There was monitored the case, when a threewheeled vehicle run in curve. Analyses were carried out in MBS software [5, 9, 27].

A technical solution relates to the front wheel fork design of an electric tricycle with two wheels on the rear axle. The goal is to improve the overturning immunity of such a vehicle, when it is run in curves.

Nowadays, steering mechanisms of three-wheeled vehicles with two wheels on the rear axle use a standard fork for the front wheel mounted on a vehicle chassis.

In current solutions of the standard three-wheeled vehicle steering, the contact point between the front wheel and the road during driving in curves is changeless. Then, the arising centrifugal force is compensated by pas- 
sengers tilting in the opposite direction, because the centre of gravity is near to the stability axis, which is given by the jumper lead of the front wheel and the external rear wheel in a curve.

Therefore, there has been occurred the requirement for the design of such front wheel suspension structure, which would ensure the overturning stability improvement of an electric tricycle and besides the conditions of the stability during braking has to be satisfied. The result of this effort is the new design of the front fork suspension of an electric tricycle.

The fundamental of our technical solution is the fact, that the front part of an electric tricycle contains the combination of rotating and shifting joints, which allows in addition to the rotary movement also the shifting movement in the lateral direction [5].

Advantages of the technical solution of the front wheel fork suspension of an electric three-wheeled vehicle are apparent from its effects, by which it expresses itself outwards. Effects of this technical solution are in that this design allows increasing the overturning immunity of an electric three-wheeled vehicle, which uses a steering of the motorcycle type. The introduced design is based on the front wheel shifting to the right side during driving in the left curve (Fig. 1) and contrariwise, i.e. always in the centrifugal force direction in order to improve the overturning stability.

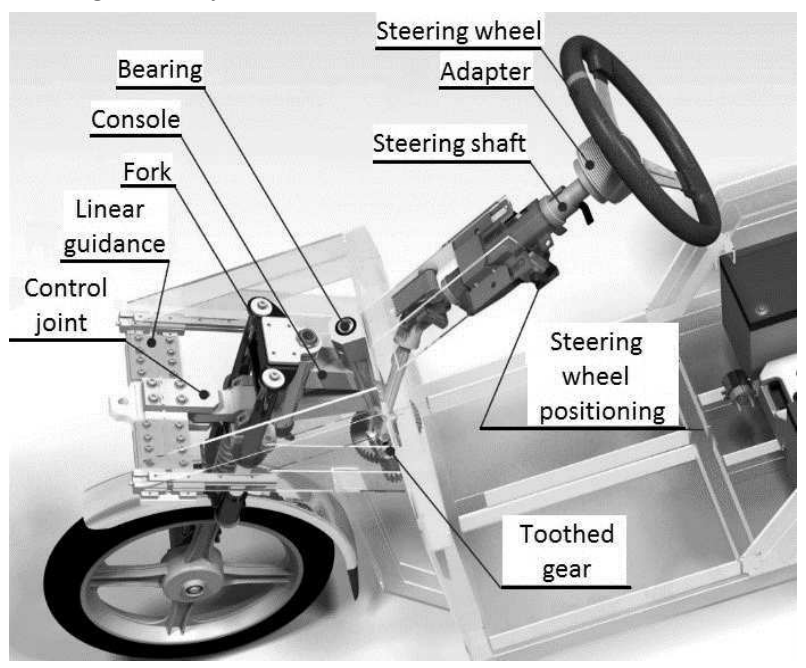

Fig. 1 Individual parts of the new steering mechanism

The described technical solution of a steering mechanism is mounted as a part of a completely new designed three-wheeled vehicle. As it is prototype of an ecological urban vehicle, its main bearing frame is made of aluminium alloy [26], which allows reduction of the total mass. An integral part of the frame design were strength analyses [17] carried out by means of Finite Element Method $[15,16,17]$.

\section{Overturning immunity of a three-wheeled ve- hicle}

In this section, geometrical relationships governing the overturning immunity of a three-wheeled vehicle with two wheels on the rear axle is driving in a curve due to a lateral acceleration are described.
This lateral acceleration is assumed to act perpendicular to the centre line of a vehicle. There are compared two technical solutions, a three-wheeled vehicle with a standard steering mechanism and with the newly designed steering mechanism of a front wheel fork, which allows except for the inevitable rotation also the lateral shifting of the front wheel $[3,14]$.
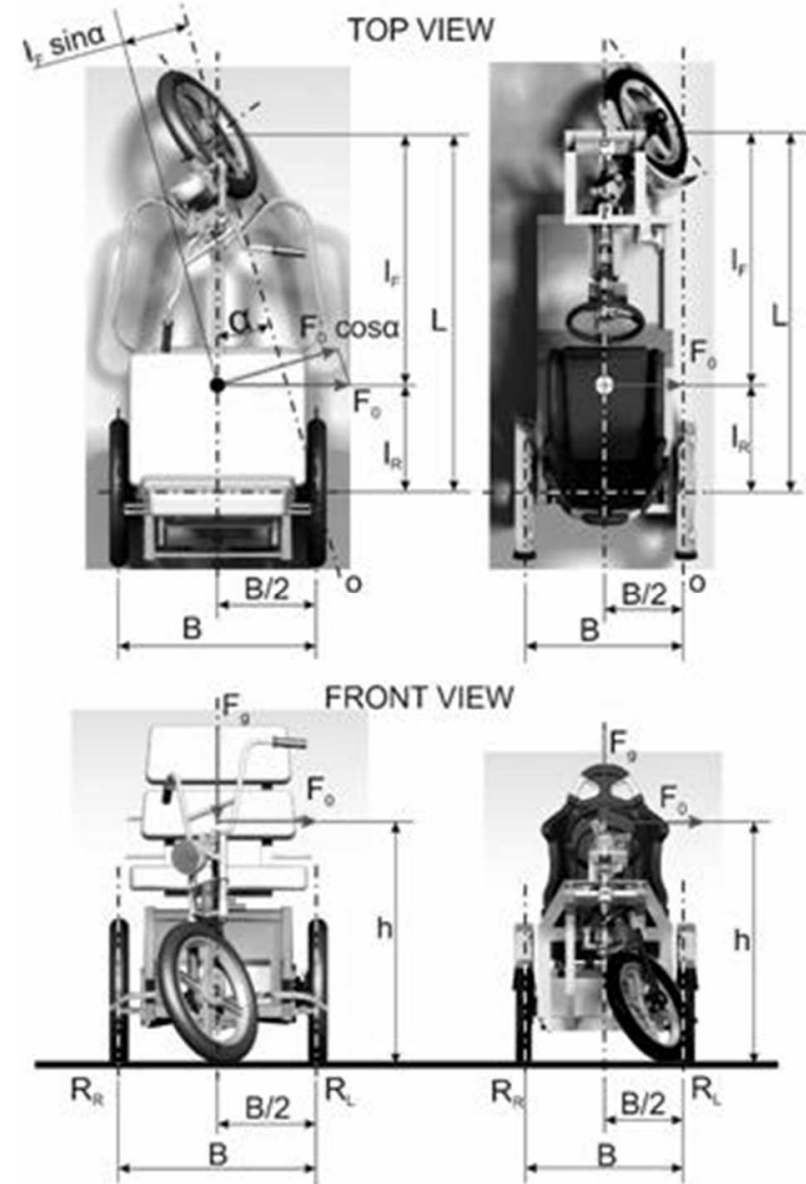

Fig. 1 Scheme of a three-wheeled vehicle with a standard steering system and with a newly designed steering system

Fig. 1 illustrates the top and front views of a threewheeled vehicle with two wheels on the rear axle, whereby the vehicle with the standard steering mechanism is shown on the left hand side and a vehicle with the new designed steering mechanism is shown on the right hand side. In this Figure, the $O$ axis represents the axis of overturning. In order the overturning immunity of a vehicle is confirmed, a negative clockwise moment about $O$ axis, as viewed from the front view of a vehicle, must exist [5]. Let's consider a three-wheeled vehicle with the standard steering mechanism (Fig. 1 left). Summing moments about the $O$ axis is given by following formulation:

$$
\begin{gathered}
\sum_{i} M_{i O} \leq 0[N \cdot m], \\
F_{o} \cdot \cos \alpha \cdot h-F_{g} \cdot l_{F} \cdot \sin \alpha \leq 0[N \cdot m],
\end{gathered}
$$

where $F_{o}[N \cdot m]$ is the force, which causes lateral acceleration (i. e. centrifugal force), $h[m]$ is the height of the centre of gravity of the three-wheeled vehicle, $l_{F}[\mathrm{~m}]$ 
is the distance of the centre of gravity from the front wheel, $\alpha\left[^{\circ}\right]$ is the angle, which is given by main dimensions of the thee-wheeled vehicle.

The formulation (1a) can be rewritten into the form:

$$
\frac{a_{o}}{g} \leq \frac{l_{F}}{h} \cdot \tan \alpha[-],
$$

where $g\left[\mathrm{~m} \cdot \mathrm{s}^{-2}\right]$ is the gravity acceleration and it represents the overturning immunity condition for a three-wheeled vehicle with the standard steering system.

By reason that the angle $\alpha$ is a function of the geometry of a considered three-wheeled vehicle, the following relation is valid:

$$
\tan \alpha=\frac{B}{2 \cdot L}[-]
$$

where $B[\mathrm{~m}]$ is the rear wheels gauge and $L[\mathrm{~m}]$ is the axle base.

If we substitute eq. (3) into eq. (2), it will give us the condition for overturning immunity of a three-wheeled vehicle with two wheels on the rear axle:

$$
\frac{a_{o}}{g} \leq \frac{B}{2 \cdot h} \cdot \frac{l_{F}}{h}[-],
$$

Let's continue with analysing of a three-wheeled vehicle with the newly designed steering mechanism. As it was mentioned above, this new technical solution of a steering mechanism allows the lateral movement (i. e. lateral shifting) of the front wheel (Fig. 1 right). Analogous to the previous case, summing moments about the $O$ axis expressed as:

$$
\begin{gathered}
\sum_{i} M_{i O} \leq 0[N \cdot m], \\
F_{o} \cdot h-F_{g} \cdot \frac{B}{2} \leq 0[N \cdot m],
\end{gathered}
$$

which we can rewrite as:

$$
\frac{a_{o}}{g} \leq \frac{B}{2 \cdot h}[-]
$$

Now we want to obtain some physical feeling for the derived conditions of the overturning immunity. Each of the above considered three-wheeled vehicles is negotiating a curve of radius $R$ to what the lateral acceleration corresponds. It is useful to express it in dependence on the vehicle forward speed $v$ and the curve radius $R$ :

$$
a_{o}=\frac{v^{2}}{R}\left[m \cdot s^{-2}\right],
$$

where $a_{o}\left[m \cdot s^{-2}\right]$ is the lateral acceleration, $v\left[m \cdot s^{-1}\right]$ is the speed of a three-wheeled vehicle, $R[\mathrm{~m}]$ is the radius of a curve.

Now we can substitute eq. (7) into the overturning immunity equations for a three-wheeled vehicle with the standard steering mechanism (eq. (4)) and also for a threewheeled vehicle with the newly designed steering mechanism (eq. (6)) the equation for the maximal vehicle's forward speed $v_{\max }$ at which the vehicle overturning occurs [1]. Then, formulation for the overturning speed for the standard steering mechanism is given by:

$$
v_{\max } \leq \sqrt{R \cdot g \cdot \frac{l_{F}}{L} \cdot \frac{B}{2 \cdot h}}\left[m \cdot s^{-1}\right],
$$

and for the newly designed steering mechanism is given by:

$$
v_{\max } \leq \sqrt{R \cdot g \cdot \frac{B}{2 \cdot h}}\left[m \cdot s^{-1}\right],
$$

In principle, the formulation (9) represents the relationship for the maximal forward speed valid for a fourwheeled vehicle.

From derived equations (8) and (9) we can identify, that $l_{F}$ is always less than $L$, the speed at which overturning of a vehicle occurs for the standard steering mechanism will always be lower than those for the corresponding the newly designed steering mechanism.

\section{Simulation computations of driving proper- ties of a three-wheeled vehicle}

In this chapter a MBS model of two variants of a three-wheeled vehicle are introduced, i. e. with the standard steering mechanism and also with the newly designed steering mechanism. The MBS models of vehicles were created in Simpack software in order to evaluate and to compare dynamic properties and driving properties (mainly overturning immunity) of both vehicles. Further, we have also compared an analytical calculation with simulation calculations of same selected parameters.

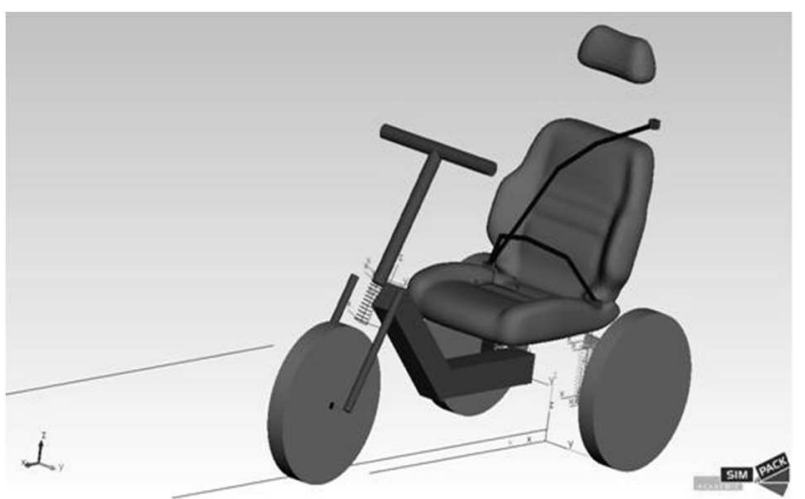

Fig. 2 MBS model of the analysed three-wheeled vehicle in Simpack software

The mentioned software enables modelling and analysing of the dynamics of road vehicles, rail vehicles [7, $8]$ and similar transport means ranging from trams [ $[10$, $11]$ to high-speed trains. It includes various tire/road contact models $[12,13]$ and wheel/rail contact model [18-22, 28], which represent an inseparable and a quite difficult modelling problem $[4,23,24]$.

In order to set up sufficiently representative virtual model, which would illustrate as faithfully as possible the reality, we have created CAD models of individual parts of both variants of three-wheeled vehicles. Based on these, we have found out mass and inertia parameters, which were input subsequently into MBS models. When we have already set up MBS models, we were able to 
found out the total mass and inertia parameters and position of the total centre of gravity needed for analytical calculations. Fig. 2 shows a simplified MBS model of the created three-wheeled vehicle and Fig. 3 shows its topo$\operatorname{logy}$.

As we wanted to investigate, how our newly designed steering mechanism improves the overturning immunity of a three-wheeled vehicle, we have chosen the driving manoeuvre in an opposing $\mathrm{S}$ curve with relatively small radius of $R=2.5 \mathrm{~m}$. Then, we performed several simulations at various speeds and we detected, when the overturning immunity is breached. As the criteria of this, we have determined the contact force between the rear internal wheel in a curve and the road. The limited case is, when the contact force equals to zero.

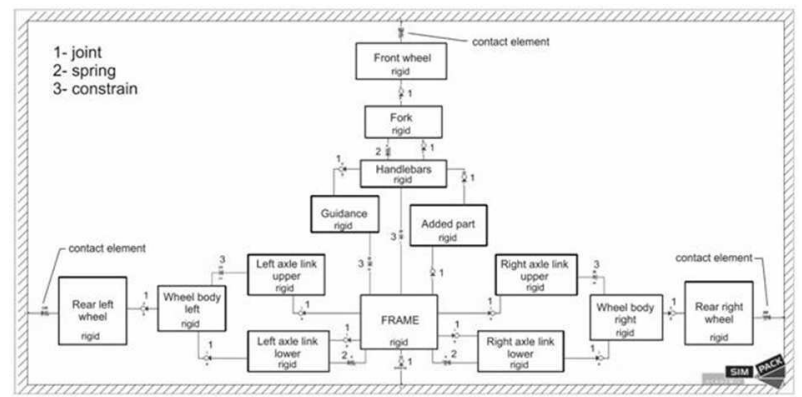

Fig. 3 Topology of MBS model in Simpack software

As we can mentioned above, the overturning immunity criteria for both the standard steering mechanism as well as the newly designed steering mechanism does not depend on the mass of the vehicle, but only on the centre of gravity position (eqs. (8), (9)) and same construction parameters of vehicles. If we consider, that required parameters resulting from vehicles design are $L=1.317 \mathrm{~m}$ and $B=0.727 \mathrm{~m}$ and the position of the centre of gravity is given by the two parameters, i.e. $h=0.43 \mathrm{~m}$ and $l_{F}=0.935 \mathrm{~m}$, then from the eq. (1), we can calculate the maximum speed of the tricycle with the standard steering mechanism for the limited case of the overturning immunity:

$$
\begin{aligned}
& v_{\max } \leq \sqrt{2.5 \cdot 9.81 \cdot \frac{0.935}{1.317} \cdot \frac{0.727}{2 \cdot 0.43}}\left[\mathrm{~m} \cdot \mathrm{s}^{-1}\right], \\
& v_{\max } \leq 3.837\left[\mathrm{~m} \cdot \mathrm{s}^{-1}\right] \doteq 13.813\left[\mathrm{~km} \cdot \mathrm{h}^{-1}\right],
\end{aligned}
$$

Results from numerical analyses are shown in Fig. 3 and Fig 4.

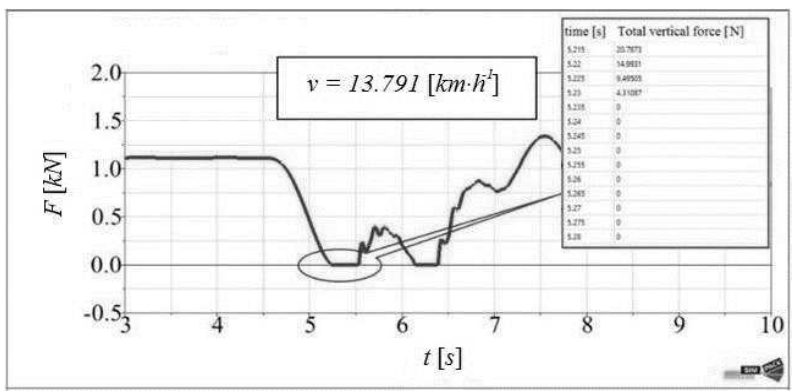

Fig. 4 Waveform of the vertical wheel force on the internal rear wheel - standard steering mechanism

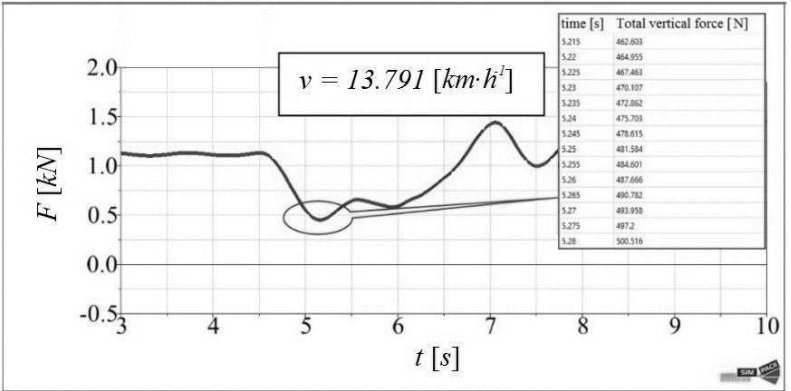

Fig. 5 Waveform of the vertical wheel force on the internal rear wheel - newly designed steering mechanism

These figures contain results for the standard steering mechanism (Fig. 3) and also for the newly designed steering mechanism (Fig. 4). There are plotted waveforms of values of total vertical wheel forces in the contact of the rear internal wheel and the road. There are also imaged number values of this parameter.

If we compare result from the analytical calculation (eq. (10)) with the result from numerical calculation (Fig. 3), we can see, the limited value of the tricycle speed of the overturning immunity is really proper found out. The little difference is caused by the fact when the tricycle is driving in a curve, the passenger's mass shifts in the lateral acceleration direction and thus the centre of gravity also shifts a bit in this direction.

Further, from results of numeral analysis of the tricycle with the new designed steering mechanism we can observe (Fig. 4), that the designed technical solution improves the overturning immunity of such a tricycle. It results from that the rear internal wheel during driving in a curve does not lose the contact with a road at all, and due to the parameters of the tricycle and driving conditions, contact forces on the rear internal wheel have sufficient values throughout the time of driving in the curve to ensure the satisfactory overturning immunity.

Based on performed calculation both analytical and numerical, we have found out, that our designed innovative steering mechanism is really able to improve the overturning immunity of a three-wheeled vehicle.

The future research in this field will be focused on further improvement of this prototype and on the development this technical solution in such a manner, that it will meet strict standards for driving safety and also for comfort for passengers [29] and it will be acceptable on the one hand from the utility point of view and on the other hand from the point of view of its simple application for the common use.

\section{Conclusion}

In this paper, a new idea of a technical solution of an innovative steering mechanism was presented. As electromobility is currently one of the key factors of reducing of the consumption of hydrocarbon fuels, the design is intended for use on an electric three-wheeled vehicle. Our steering system is designed in order to increase overturning immunity of a three-wheeled vehicle in curves, what means, that driving in this vehicle is safer and also more comfortable. As the essential advantage of this 
solution there can be considered the fact, that it is purely based on the mechanical principle.

From calculation we found out, that our technical approach to a steering mechanism system allows increasing the overturning immunity of a tricycle. It was proved based on the comparison of analyses of driving these three-wheeled vehicle in curves at the same driving conditions (speed, curve radius). For implementation of this innovative steering mechanism there is necessary to perform same technical modification in such way, that the operation of such an electric tricycle will be safe, comfortable and effective.

\section{Acknowledgement}

This work was supported by the Cultural and Educational Grant Agency of the Ministry of Education of the Slovak Republic in the project No. KEGA 007ŽU4/2017: Modernization of the Vehicle and engines study program.

\section{References}

[1] BARAN, P., BREZANI, M., KUKUCA, P., STASTNIAK, P. (2017). Basic dynamical analysis and comparison of balancing systems of nonconventional piston machine FIK. In: Procedia Engineering, Vol. 192, pp. 34-39.

[2] BARAN, P., STASTNIAK, P., KUKUCA, P., BREZANI, M. (2018). Analysis of kinematic features in non-conventional piston device with wobble board. In: Manufacturing Technology, Vol. 18, No. 2, pp. 179-184.

[3] BArote, J., DARling, J., Plummer, A. (2015). Lateral dynamics simulation of a threewheeled tilting vehicle. In: Proceedings of the Institution of mechanical Engineers, Part D: Journal of Automobile Engineering 229, 342-356 (2015)

[4] CHALUPA, M. (2015). The using of vehicle moving simulation to proposal of construction work. In: Manufacturing Technology, Vol. 15, No. 5, pp. 845-850.

[5] DIZO, J., BARTA, D., BLATNICKY, M. (2018). Improvement driving characteristics of electric tricycle. In: Proceedings of Engineering for Rural Development 17, pp. 2123-2128.

[6] FALENDYSH, A., KHARLAMOV, P., KLETSKA, O., VOLODARETS, O. (2016). Calculation of the parameters of hybrid shunting locomotive. In: Transportation Research Procedia, Vol. 14, pp. 665-671.

[7] GERLICI, J., GORBUNOV, M., NOZHENKO, O., PISTEK, V., KARA, S., LACK, T., KRAVCHENKO, K. (2017). About creation of bogie of the freight car. In: Communications - Scientific Letters of the University of Zilina, Vol. 19, No. 2, pp. 29-35.
[8] GERLICI, J., LACK, T. (2009). Iterative method for railway wheel profile design. In: Komunikacie, Vol. 11, No. 2, pp. 49-56.

[9] HAUSER, V., NOZHENKO, O., KRAVCHENKO, K., LOULOVA, M., GERLICI, J., LACK, T. (2017). Impact of three axle boxes bogie to the tram behaviour when passing curved track. In: Procedia Engineering, Vol. 192, pp. 295-300.

[10] HAUSER, V., NOZHENKO, O., KRAVCHENKO, K., LOULOVA, M., GERLICI, J., LACK, T. (2017). Proposal of a mechanism for setting bogie wheelset to radial position while riding along track curve. In: Manufacturing Technology, Vol. 17, No. 2, pp. 286-192.

[11] HAUSER, V., NOZHENKO, O., KRAVCHENKO, K., LOULOVA, M., GERLICI, J., LACK, T. (2017). Proposal of a steering mechanism for tram bogie with three axle boxes. In: Procedia Engineering, Vol. 192, pp. 289-294.

[12] ISTENIK, R., BARTA, D., MUCHA, W. (2004). Influence of the wheels on the automobile dynamics. In: Komunikacie, Vol. 6, No. 1, pp. 26-28.

[13] JONAK, M., KASPAREK, J. (2015). Discrete computational model of a tire. In: Proceedings of $19^{\text {th }}$ International Scientific Conference on Transport Means, TRANSPORT MEANS 2015, pp. 341-344.

[14] JUBIN, J. A., JAYBAL, K. (2016). Rollover dynamics of a narrow tilting three-wheeled vehicle. In: MATEC Web of Conferences, Vol. 51.

[15] KLIMENDA, F., RYCHLIKOVA, L., HUSAR, S., SOUKUP, J. (2017). Dissemination of waves in thin plates. In: Manufacturing Technology, Vol. 17, No. 6, pp. 863-869.

[16] KLIMENDA, F., SKOCILASOVA, B. (2015). Rollers vibration of pipe conveyor. In: Manufacturing Technology, Vol. 15, No. 6, pp. 991-995.

[17] KURCIK, P., HARUSINEC, J., STRAZOVEC, P., PAVLIK, A. (2018). Strength analysis of an E3-kolka frame design (In Slovak). In: Proceedings of $5^{\text {th }}$ International Conference for young scientific workers EVM 2018, pp. 61-69.

[18] LACK, T., GERLICI, J. (2005). Contact area and normal stress determination on railway wheel/rail contact. In: Komunikacie, Vol. 7, No. 2, pp. 38-45.

[19] LACK, T., GERLICI, J. (2012). Modified strip method utilisation for wheel/rail contact stress evaluation. In: Proceedings of $9^{\text {th }}$ International Conference on Contact Mechanics and Wear of Rail/Wheel Systems, CM 2012, Chengdu, China, pp. 87-89. 
[20] LACK, T., GERLICI, J. (2009). Railway wheel and rail roughness analysis. In: Komunikacie, Vol. 11, No. 2, pp. 41-48.

[21] LACK, T., GERLICI, J. (2013). Wheel/rail contact stress evaluation by means of the modified strip method. In: Komunikacie, Vol. 15, No. 3, pp. 126-132.

[22] LACK, T., GERLICI, J. (2014). Wheel/rail tangential contact stress evaluation by means of the modified strip method. In: Communications - Scientific Letters of the University of Zilina, Vol. 16, No. 3a, pp. 33-39.

[23] LOULOVA, M., SUCHANEK, A., HARUSINEC, J. (2017). Evaluation of the parameters affecting passenger riding comfort of a rail vehicle. In: Manufacturing Technology, Vol. 17, No. 2, pp. 224-231.

[24] MANUROVA, M., SUCHANEK, A. (2016). The analysis of a rail vehicle with a tilting bogie. In: Manufacturing Technology, Vol. 16, No. 5, pp. 1020-1027.

[25] MRUZEK, M., GAJDAC, L., KUCERA, L., BARTA, D. (2016). Analysis of parameters influencing electric vehicle range. In: Procedia Engineering, Vol. 134, pp. 165-174.
[26] PASTIRCAK, R., SCURY, J., MORAVEC, J. (2017). The effects of pressure during the crystallization on properties of the alsi12 alloy. In: $A r$ chives of Foundry Engineering, Vol. 17, No. 3, pp. 103-106.

[27] SAPIETOVA, A., BUKOVAN, J., SAPIETA, M., JAKUBOVICOVA, L. (2017). Analysis and implementation of input load effects on an air compressor piston in MSC.ADAMS. In: Procedia Engineering 177, 554-561 (2017)

[28] SMETANKA, L., STASTNIAK, P. (2017). Analysis of contact stresses of theoretical and worn profile by using computer simulation. In: Manufacturing Technology, Vol. 17, No. 4, pp. 580-585.

[29] SVOBODA, M., SOUKUP, J., JELEN, K., KUBOVY, P. (2015). Effect of impacts on human head. In: Manufacturing Technology, Vol. 15, No. 2, pp. 226-231.

[30] TARTAKOVSKY, E., FALENDYSH, A., ZINKIVSKYI, A., MIKHEEV, S. (2015). Refining the models of performing service tests of upgraded locomotives. In: EasternEuropean Journal of Enterprise Technologies, Vol. 2, No. 3, pp. 2631 . 